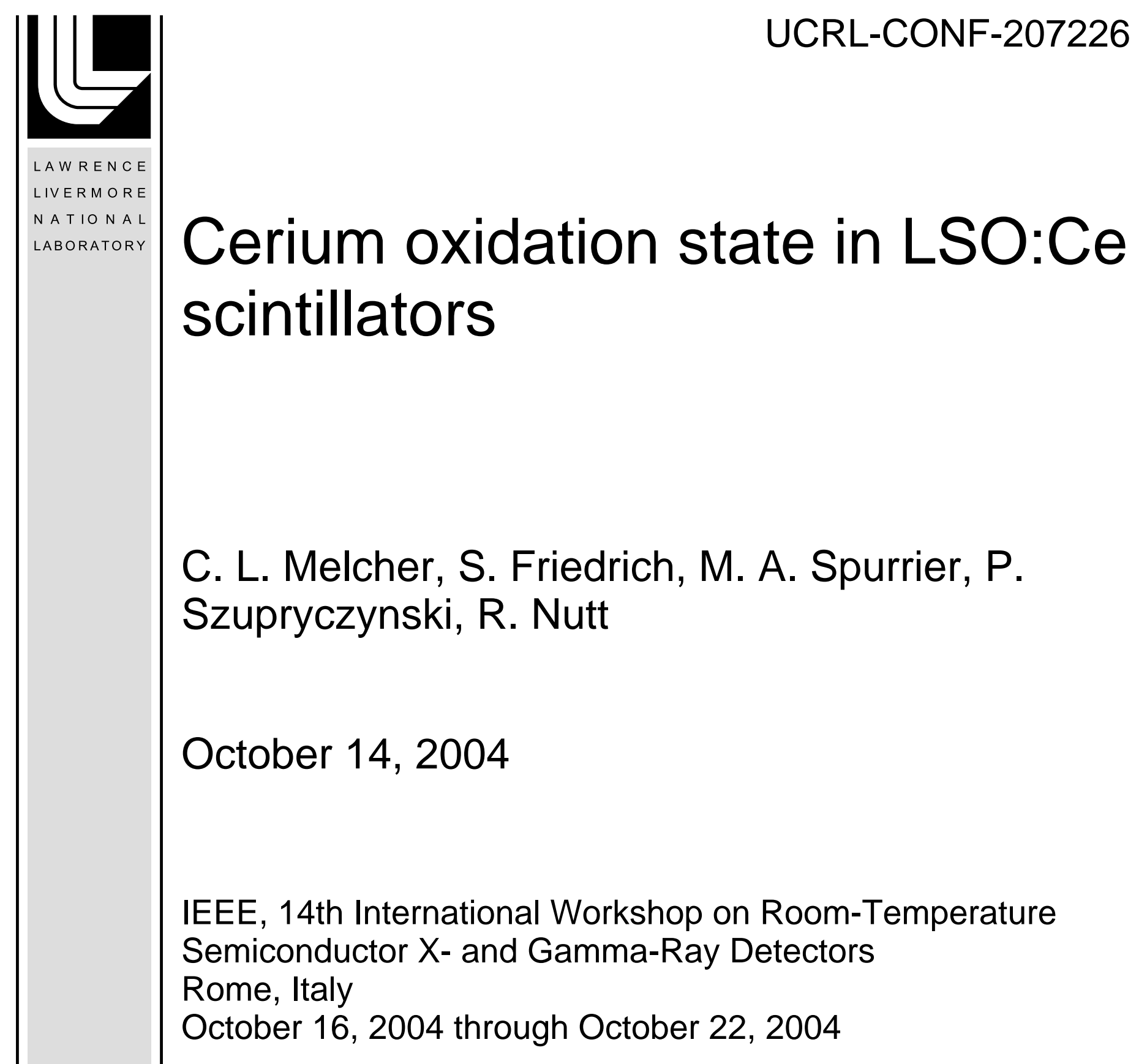


This document was prepared as an account of work sponsored by an agency of the United States Government. Neither the United States Government nor the University of California nor any of their employees, makes any warranty, express or implied, or assumes any legal liability or responsibility for the accuracy, completeness, or usefulness of any information, apparatus, product, or process disclosed, or represents that its use would not infringe privately owned rights. Reference herein to any specific commercial product, process, or service by trade name, trademark, manufacturer, or otherwise, does not necessarily constitute or imply its endorsement, recommendation, or favoring by the United States Government or the University of California. The views and opinions of authors expressed herein do not necessarily state or reflect those of the United States Government or the University of California, and shall not be used for advertising or product endorsement purposes. 


\title{
Cerium oxidation state in LSO:Ce scintillators
}

\author{
Charles L. Melcher, Senior Member IEEE, Stephan Friedrich, Merry A. Spurrier,
}

\author{
Piotr Szupryczynski, Ron Nutt Fellow IEEE
}

\begin{abstract}
Trivalent cerium ions form the luminescence centers in several important families of scintillation materials including the rare earth oxyorthosilicates, pyrosilicates, and aluminates. When comparing the experimentally determined scintillation properties of cerium-doped scintillators to theoretical models of scintillation mechanisms, there is often speculation regarding the fraction of the total cerium that exists in the radiative trivalent charge state $\left(\mathrm{Ce}^{3+}\right)$ rather than the non-radiative tetravalent state $\left(\mathrm{Ce}^{4+}\right)$. Until now, however, no technique has been developed to quantitatively measure both $\mathrm{Ce}^{3+}$ and $\mathrm{Ce}^{4+}$. We report here for the first time direct measurements of $\mathrm{Ce}^{3+}$ and $\mathrm{Ce}^{4+}$ in $\mathrm{Lu}_{2} \mathrm{SiO}_{5}$ :Ce scintillators. Synchrotron radiation was used to measure the $x$-ray absorption on the $M_{4}$ and $M_{5}$ edges of $C e$, and the results were compared to model samples of $\mathrm{Ce}^{3+}\left(\mathrm{Ce}_{2} \mathrm{O}_{3}\right)$ and $\mathrm{Ce}^{4+}\left(\mathrm{CeO}_{2}\right)$ which provided clear signatures of the two charge states. The spectra were obtained with a high-resolution superconducting tunnel junction spectrometer on beamline 4.0.2 at the Advanced Light Source synchrotron at Lawrence Berkeley National Laboratory. The results clearly show $100 \% \mathrm{Ce}^{3+}$, independent of light yield and sample coloration. Therefore, energy migration to the luminescence centers appears to be the determining factor in the scintillation efficiency of these samples, rather than variations in the $\mathrm{Ce}^{3+} / \mathrm{Ce}^{4+}$ ratio.
\end{abstract}

\section{INTRODUCTION}

As cerium-doped scintillators have increased in significance, considerable effort has gone into the characterization of the host material properties and the investigation of scintillation mechanisms that include the excitation and emission of the $\mathrm{Ce}$ luminescence centers. Although $\mathrm{Ce}$ can exist in either the trivalent $\left(\mathrm{Ce}^{3+}\right)$ or tetravalent $\left(\mathrm{Ce}^{4+}\right)$ state, it is well understood that only trivalent $\mathrm{Ce}$ gives rise to luminescence emission. In order to compare experimentally determined scintillation characteristics with models of the scintillation mechanism, one usually needs to know the fraction of total $\mathrm{Ce}$ in the crystal that exists in the trivalent state. Optimization of the crystal growth process also relies heavily on knowledge of the $\mathrm{Ce}$ charge state.

Charles L. Melcher, Merry A. Spurrier, Piotr Szupryczynski and Ron Nutt are with CTI Molecular Imaging, Inc., 810 Innovation Dr., Knoxville, TN 37932 USA (telephone: 865-218-2000).

Stephan Friedrich is with the Advanced Detector Group at Lawrence Livermore National Laboratory, 7000 East Ave, L-270, Livermore, CA 94550.
The relative populations of $\mathrm{Ce}^{3+}$ and $\mathrm{Ce}^{4+}$ in a scintillator are often estimated from analyses of the total $\mathrm{Ce}$ and the $\mathrm{Ce}^{3+}$ since a direct measurement of $\mathrm{Ce}^{4+}$ has been elusive. The total Ce can be measured by various techniques including glow discharge mass spectroscopy (GDMS), inductively coupled plasma mass spectroscopy (ICPMS), and X-ray fluorescence (XRF). However, in our experience, the results usually have uncertainties in excess of $20 \%$ and often times much greater due, at least in part, to the lack of reliable calibration standards in the matrix of interest. $\mathrm{Ce}^{3+}$ can be observed by UV-visible spectroscopy, but it is difficult to determine the concentration quantitatively with good precision again due to the lack of good calibration standards. Since $\mathrm{Ce}^{4+}$ is not optically active, it cannot be observed directly.

X-ray absorption spectroscopy (XAS) can be used to precisely determine the electronic energy levels of elements, including small shifts in these levels due to the oxidation state of the ion. A highly monochromatic X-ray beam is scanned in energy through the region of the electron binding energy and the resulting absorption is measured. Rather than measure absorption via attenuation of the incident beam, quantities that are directly proportional to absorption are more commonly utilized. Concentrated compounds such as the $\mathrm{Ce}^{3+}{ }_{2} \mathrm{O}_{3}$ and $\mathrm{Ce}^{4+} \mathrm{O}_{2}$ models compounds in the current study are best measured via the total electron yield (TEY) where photoelectrons and Auger electrons emitted by the sample are measured by a channeltron. Dilute samples such as the Cedoped scintillator crystals studied here are best measured via the partial fluorescence yield (PFY) where a high resolution detector selects fluorescence only from the element of interest and thus provides much improved sensitivity.

\section{EXPERIMENTAL}

Single crystal boules of Ce-doped $\mathrm{Lu}_{2} \mathrm{SiO}_{5}$ (LSO:Ce) were grown from the melt via the Czochralski technique in inductively heated iridium crucibles [Melcher 1995]. The $\mathrm{Lu}_{2} \mathrm{O}_{3}, \mathrm{SiO}_{2}$, and $\mathrm{CeO}_{2}$ starting materials were at least $99.99 \%$ pure. Crystal growth was initiated with seed crystals, and was controlled via an automated system that used the derivative of the crystal weight as the process variable. Samples with dimensions of $3 \mathrm{~mm} \times 4 \mathrm{~mm} \times 1 \mathrm{~mm}$ were cut with a diamond saw.

Prior to measurement of the scintillation light yield, the samples were stored in the dark for at least 24 hours to eliminate the thermoluminescence emission that is stored upon 
exposure to white light. The scintillation light yield was measured by placing the crystal directly onto a Hamamatsu R877 photomultiplier tube. The crystal was covered with a loose fitting Teflon cap to enhance the light collection efficiency. A $10 \mu \mathrm{Ci}{ }^{137} \mathrm{Cs}$ source was located $\sim 15 \mathrm{~mm}$ from the crystal surface. The natural background spectrum coming from $\mathrm{Lu}^{176}$ beta decay was minimal due to the small sample size and was not subtracted. The light output was reproducible to within $+/-5 \%$.

X-ray absorption spectra on the $\mathrm{Ce} \mathrm{M}_{4}$ and $\mathrm{M}_{5}$-edges were taken at beam line 4.0.2 of the Advanced Light Source synchrotron at Lawrence Berkeley National Laboratory [Young 2002]. The synchrotron provides a flux of $\sim 10^{12}$ photons/sec, and the beamline monochromator has an energy resolution of $\sim 0.15 \mathrm{eV}$ at a slit setting of $20 \mu \mathrm{m} / 20 \mu \mathrm{m}$. This is well below the natural line width of the X-ray absorption features. The two absorption edges at $\sim 884$ and $\sim 902 \mathrm{eV}$ correspond to dipole-allowed transitions between the $\mathrm{Ce} 3 \mathrm{~d}_{3 / 2}$ and the $3 \mathrm{~d}_{5 / 2}$ core levels and the $4 \mathrm{f}$ valence band as shown in Figure 1.

N

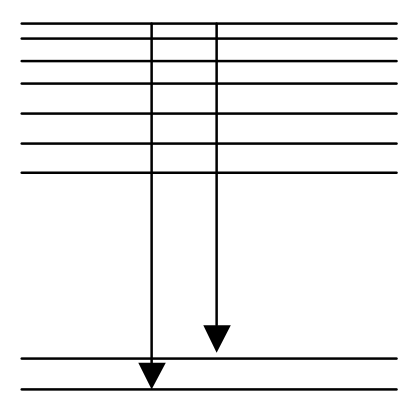

$\mathrm{N}_{7} 4 \mathrm{f}_{5 / 2}$

$\cdot$

$\mathrm{N}_{1} 4 \mathrm{~s}$

M

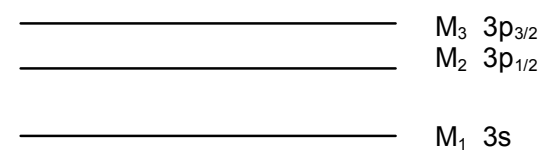

Fig. 1. Energy levels of cerium. The $\mathrm{M}_{4}$ and $\mathrm{M}_{5}$ absorption edges shift as a function oxidation state. The binding energies of these levels are shown for metallic cerium [Cardona and Ley, 1978]. The arrows indicate fluorescence emission.

The $\mathrm{Ce}^{3+}{ }_{2} \mathrm{O}_{3}$ and $\mathrm{Ce}^{4+} \mathrm{O}_{2}$ model compounds were obtained from commercial suppliers in powder form. Since $\mathrm{Ce}^{3+}{ }_{2} \mathrm{O}_{3}$ oxidizes in air, this sample was stored in argon with the exception of a few minutes air exposure during grinding and loading into the vacuum chamber. No special precautions were needed for the $\mathrm{Ce}^{4+} \mathrm{O}_{2}$ sample. The model compounds were measured by total electron yield with a channeltron electron multiplier.

Absorption spectra of Ce dopants in LSO:Ce single crystals were measured by partial fluorescence yield using a superconducting tunnel junction spectrometer and gating on the Ce M fluorescence [Friedrich 2002]. The detector was a 9element array of $\mathrm{Nb}-\mathrm{Al}-\mathrm{AlOx}-\mathrm{Al}-\mathrm{Nb}$ tunnel junctions cooled to $0.1 \mathrm{~K}$ by a two-stage adiabatic demagnetization refrigerator and provided $\sim 15 \mathrm{eV}$ resolution to isolate the fluorescence from the Ce dopants from other more abundant elements in the samples. At the absorption edges, the incident energy was increased in $0.1 \mathrm{eV}$ steps with an integration time of $10 \mathrm{~s}$ at each energy, with larger $0.5 \mathrm{eV}$ steps in the featureless regions of the spectrum. Spectra were normalized by the incident photon flux, a step-function background was subtracted to account for electron excitation into the continuum, and the absorption signal at the $\mathrm{M}_{5}$-edge was set to unity. The energy was calibrated by setting the $\mathrm{M}_{5}$-edge of $\mathrm{Ce}_{2} \mathrm{O}_{3}$ to $883.8 \mathrm{eV}$. The calibration was constant for each beam time over the two days of data acquisition, but shifted by $\sim 1 \mathrm{eV}$ during the two months between the two beam times when these data were taken.

\section{RESULtS}

\section{A. Scintillation properties}

Following several years of development for application in positron emission tomography (PET), LSO:Ce scintillator crystals have reached a rather mature stage where large quantities of crystals are produced with uniformly high light yield and consistent decay time [Melcher 2003]. Occasionally, however, unintentional deviations in the growth process or contamination of raw materials may result in crystals with low light yield or yellow discoloration. Such cases provide an opportunity to study the various factors that affect the scintillation performance. For this investigation we chose samples that represented a range of light yield in order to test the hypothesis that differences in light yield result, at least in part, from variations in the $\mathrm{Ce}^{3+} / \mathrm{Ce}^{4+}$ ratio in the crystals. Samples LSO-1, LSO-3, and LSO-5 were colorless crystals with high light yield ( $>25,000 \mathrm{ph} / \mathrm{MeV})$. Sample LSO-2 was also colorless, but had an anomalously low light yield that was less than half of the other crystals. Sample LSO-4 had a yellow discoloration but with only slightly lower light yield than the best crystals. The samples are summarized in Table 1 along with the light yield measured under gamma-ray excitation from a ${ }^{137} \mathrm{Cs}$ source.

TABLE 1. LIGHT YIELD OF $3 \times 4 \times 1$ MM LSO:CE SINGLE CRYSTAL SAMPLES.

\begin{tabular}{lccc}
\hline Designation & $\begin{array}{c}\text { Ce conc. } \\
(\mathbf{\%})^{*}\end{array}$ & Color & $\begin{array}{c}\text { Light yield } \\
(\mathbf{p h} / \mathbf{M e V})\end{array}$ \\
\hline LSO - 1 & 0.2 & None & 25,000 \\
LSO - 2 & 0.2 & None & 11,900 \\
LSO - 3 & 0.2 & None & 28,500 \\
LSO - 4 & 0.2 & Yellow & 24,200 \\
LSO - 5 & 0.2 & None & 25,800 \\
\hline
\end{tabular}

${ }^{*}$ Ce concentration in the melt from which the crystal was grown.

\section{B. X-ray absorption}

Powdered samples of $\mathrm{Ce}_{2} \mathrm{O}_{3}$ and $\mathrm{CeO}_{2}$ were used as model samples of $\mathrm{Ce}^{3+}$ and $\mathrm{Ce}^{4+}$ respectively. The X-ray absorption spectra of these samples are shown in Figure 2 where one sees two sets of sharp lines for each sample due to transitions 
between the $3 \mathrm{~d}$ core levels and the $4 \mathrm{f}$ valence level. For $\mathrm{Ce}^{4+}$ the lines are shifted to significantly higher energies compared to $\mathrm{Ce}^{3+}$ due to reduced electron screening and consequently tighter binding. The fine structure in the absorption features reflects differences in the site symmetry and ligand field strength of the cubic $\mathrm{CeO}_{2}$ and the hexagonal $\mathrm{Ce}_{2} \mathrm{O}_{3}$. Thanks to the intrinsically narrow lines of these inner shell transitions and the energy resolution of the measurement system, the patterns for the two samples are easily distinguished from each other.

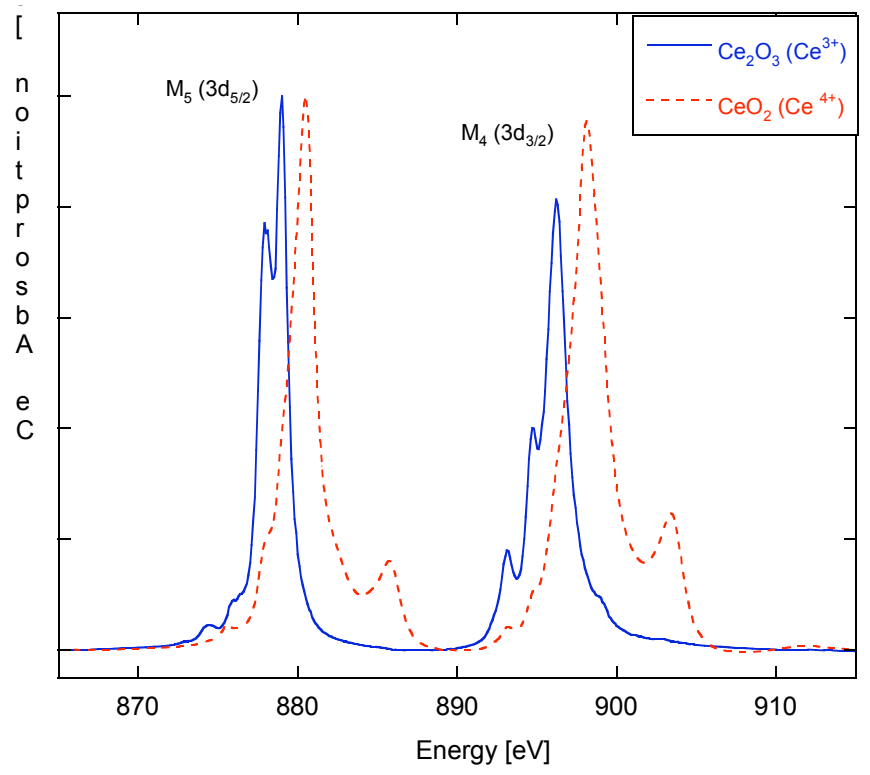

Fig. 2. X-ray absorbance of $\mathrm{Ce}^{3+}$ and $\mathrm{Ce}^{4+}$ model samples showing the shift in the $\mathrm{M}_{4}$ and $\mathrm{M}_{5}$ edges due to the difference in screening by the outer electron shells.

Figure 3 shows the absorption spectrum for sample LSO-1 superimposed on the model compounds. The lines in the LSO1 spectrum match the $\mathrm{Ce}^{3+}$ model extremely well, with virtually no evidence of $\mathrm{Ce}^{4+}$, thus confirming that essentially all of the cerium in this sample is in the trivalent state. This might be expected for this particular sample since its scintillation properties are quite good. The small differences in the fine structure of the Ce absorption edges between LSO-1 and $\mathrm{Ce}_{2} \mathrm{O}_{3}$ result from the different symmetry and ligand fields. In LSO-1, i.e. in cerium doped LSO, the cerium resides at two tetragonal sites with 6 and 7 fold oxygen coordination, compared to the hexagonal structure of $\mathrm{Ce}_{2} \mathrm{O}_{3}$.

The X-ray absorption spectra for all four of the cerium doped LSO crystals are shown in Fig. 4. Clearly all four spectra are virtually identical to the LSO-1 spectrum shown in Fig. 3, displaying little evidence of $\mathrm{Ce}^{4+}$. This is surprising in the case of LSO-2 (low light yield) and LSO-4 (yellow color) since low light output and yellow color are often assumed to suggest the presence of $\mathrm{Ce}^{4+}$. Apparently this is not the explanation for the scintillation properties of these two crystals.

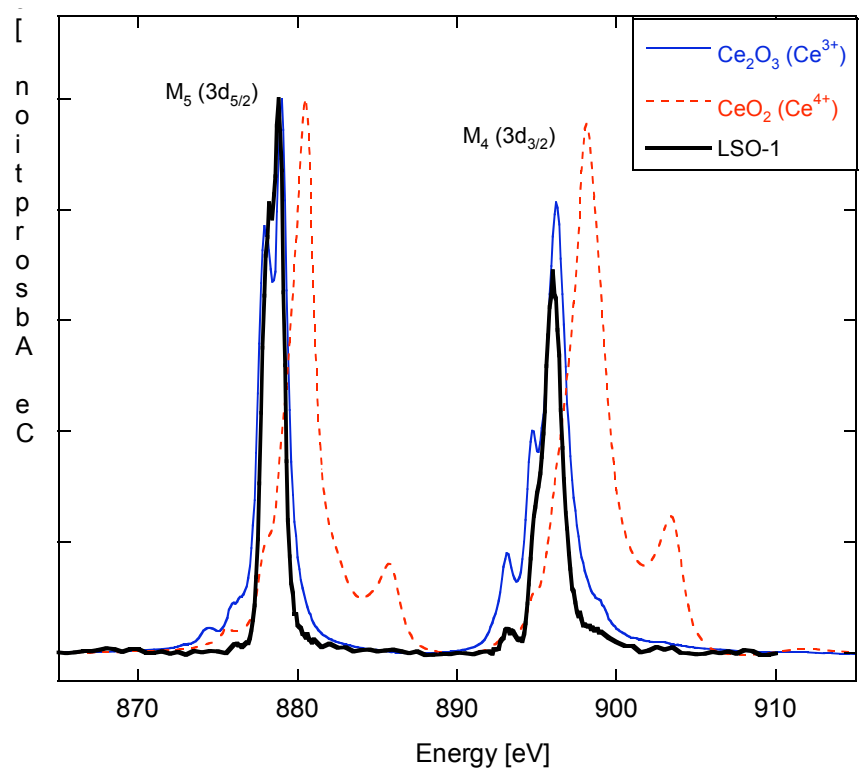

Fig. 3. X-ray absorbance spectra comparing the $\mathrm{Ce} \mathrm{M}_{4}$ and $\mathrm{M}_{5}$ edges in LSO:Ce to the $\mathrm{Ce}^{3+}$ and $\mathrm{Ce}^{4+}$ model compounds.

Surprisingly, all of the samples in Table 1 displayed X-ray absorption spectra that were nearly identical to the LSO:Ce spectrum in Figure 3. This is a significant result because it means that different proportions of $\mathrm{Ce}^{3+}$ and $\mathrm{Ce}^{4+}$ cannot explain the differences in light yield as well as the yellow coloration of some samples. Thus, it appears likely that variations in the energy migration process, possibly due to different populations of charge carrier traps, are responsible for the differences in light yields of rare earth oxyorthosilicate scintillators.

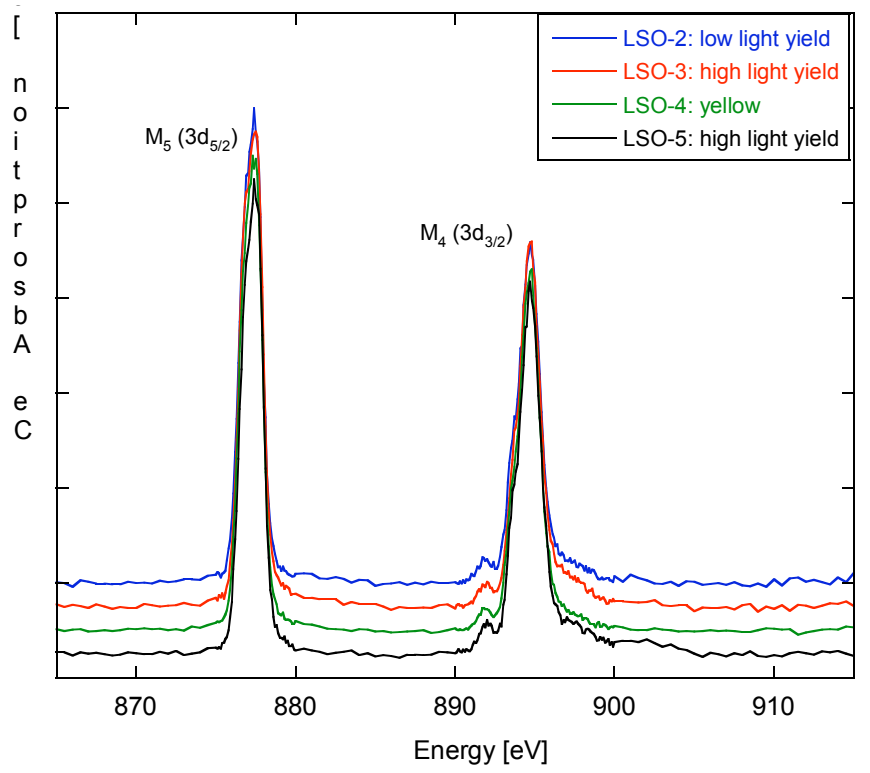

Fig. 4. X-ray absorbance spectra of LSO:Ce crystals with both low and high light yield and one crystal exhibiting a yellow discoloration. The spectra are displaced vertically for clarity. 


\section{SUMMARY AND CONCLUSION}

To our knowledge, this is the first direct and simultaneous measurement of both $\mathrm{Ce}^{3+}$ and $\mathrm{Ce}^{4+}$ in scintillators. The high resolution of the beamline and of the superconducting tunnel junction detector array allowed distinct signatures for $\mathrm{Ce} 3+$ and $\mathrm{Ce}^{4+}$ to be observed. The differences in the $\mathrm{M}_{4}$ and $\mathrm{M}_{5}$ absorption edges result from shifts in the binding energies due to the oxidation state of $\mathrm{Ce}$.

When comparing experimentally determined scintillation properties to the predicted scintillation efficiency of theoretical models, the quantum efficiency of the luminescence centers must be determined. Since cerium may exist as either $3+$ or $4+$, but only the $3+$ state results in scintillation emission, it is crucial to know the relative proportions of the two states. In addition, knowledge of the relative populations of the two oxidation states will aid in the optimization of crystal growth processes to maximize the scintillation efficiency.

Surprisingly, all of the samples investigated in this study, whether high or low light yield, colorless or yellow, contained $\mathrm{Ce}^{3+}$ only. No evidence of $\mathrm{Ce}^{4+}$ was observed, despite that fact that the $\mathrm{Ce}^{4+} \mathrm{O}_{2}$ model compound demonstrated that $\mathrm{Ce}^{4+}$ would be easily observable if it were indeed present. Evidently, the low light yield of one crystal and the yellow coloration of another crystal must be explained by other phenomena.

Although we did not attempt to quantitatively determine the sensitivity of the XAS technique for detecting either $\mathrm{Ce}^{3+}$ or $\mathrm{C}^{4+}$, we estimate that less than $5 \%$ of either species should be readily detectable at typical dopant concentrations.

\section{ACKNOWLEDGMENTS}

We thank Owen Drury and Simon J. George for assistance with the XAS measurements. This work was performed under the auspices of the U.S. Department of Energy by University of California Lawrence Livermore National Laboratory under contract No. W-7405-Eng-48.

\section{REFERENCES}

[Young 2002]

[Friedrich 2002]

[Melcher 1995]

[Melcher 2003]

[Cardona and Ley, 1978] 